\title{
Research on the Architecture of the Networked Function Interface Based on Component
}

\author{
Li Wanling, Chen Peng, Liu Yaozhou \\ Troops 63908 PLA \\ Shijiazhuang, China \\ 759934614@qq.com
}

Keywords: function interface; module structure; network.

\begin{abstract}
To resolve using TPS, upgrading and replacing instrument conveniently, referenced from the module with IVI, IVI-MSS and IVI-Signal Interface, network function interface module based on module technology was presented in this paper. The method avoided the operation to the detail instrument, and ensured the test and the instruments independent.
\end{abstract}

\section{Introduction}

With the continuous development of automatic testing and network technology, the research and application of automatic testing system based on network is highly focused in region. To meet this change, making full use of network media, implementing expert resources sharing, technical supporting, collaborative testing, diagnosis on line, breaking through the limits of time and space, it's bound to be a trend constructing automatic test system based on network. If ATS is upgraded, TPS needs developing and compiling again because of it's upgrade and update. It's more complex and difficult for developing and maintaining in view of network automatic testing system. Therefore, it is more important whether to use TPS or not, or weather to upgrade and replace instrument conveniently or not.

Based on this, the paper presents an idea with network function interface module based on module technology. The basic principle is referenced from the module with IVI, IVI-MSS and IVI-Signal Interface, and it will be packaged on the further. It generates file .EXE, being packaged by the form of remote process external unit. Not only it is called by local computer, but also by remote terminal.

\section{Module Structure}

No matter how complex test system is, in terms of system, that the basic type on test signal or pumping signal is always finite. But, to a certain kind of a signal specifically, no matter by what instruments or modules to test or drive is, it's the same on the function. For example, the direct current signal voltage is tested by $\mathrm{A} / \mathrm{D}$ or DMM, it's unnecessary to learn the specific implementation details and instrument control process. You can write the test process making function set and measured nodes adopted explicitly, to the tester. The instrument can be packaged on sorting according to the demand with signal's test or excitation function. And you can ignore the implicated details of the specific function of each instrument. No matter what kind of instrument is, so long as has the same function, the same function driver interface does. (For example, it's the same as function driver interface with digital multimeter and measuring voltage module.) Establishing the standard instrument function driver module library, the function interface abstracted fatherly by the common function among all the functions of the instrument driving module is provided to the user. It's achieved in the internal function interface with instrument automatic selection and signal channel connection. Which instrument operation is called specifically, it is a decision by the need of the functional interface test and system test resource allocation dynamic. 
The module of the network function interface based on component borrows ideas from the basic principle of IVI, IVI-MSS and IVI-Signal Interface module. The test procedure should turn it's face to the signal UUT, but not face the equipment operating directly. The function interface layer is set up between test procedure and specific instrument control. It avoided the operation to the detail instrument with the procedure, isolating instrument control and test procedure completely. Certainly, the instrument has no effect on the test procedure. This will ensure the test and the instruments independent. And then, it's very easy to upgrade test procedure and instrument. The structure with network function interface module based on module technology is shown in figure 1.It is composed of function agent, resource management engine, driving module and signal channel control module four parts.

The function agent module of function interface receives the virtual resource need submitted by the test procedure, transforms the virtual resource need into the corresponding entry and starts the resource management engine .For the following operation in turn: Firstly, it should seek the function resource conforming to the test requirement from equipment module library on priority level, and locate the virtual resource to the real resource. Afterwards, it should map the UUT port to the switch resource output port in accordance with the transfer module of adapter signal, examine the switch element is idle or not to electrical connections between UUT signal port and instrument port, execute connection algorithm of UUT port and signal port find out the optimal signal channel path call the signal channel control module and realize the connection control of signal channel. Lastly it can achieve the set on the instrument property referenced with the real resource, by the method and property of the signal, and execute test or drive calling the specific instrument function drive module.

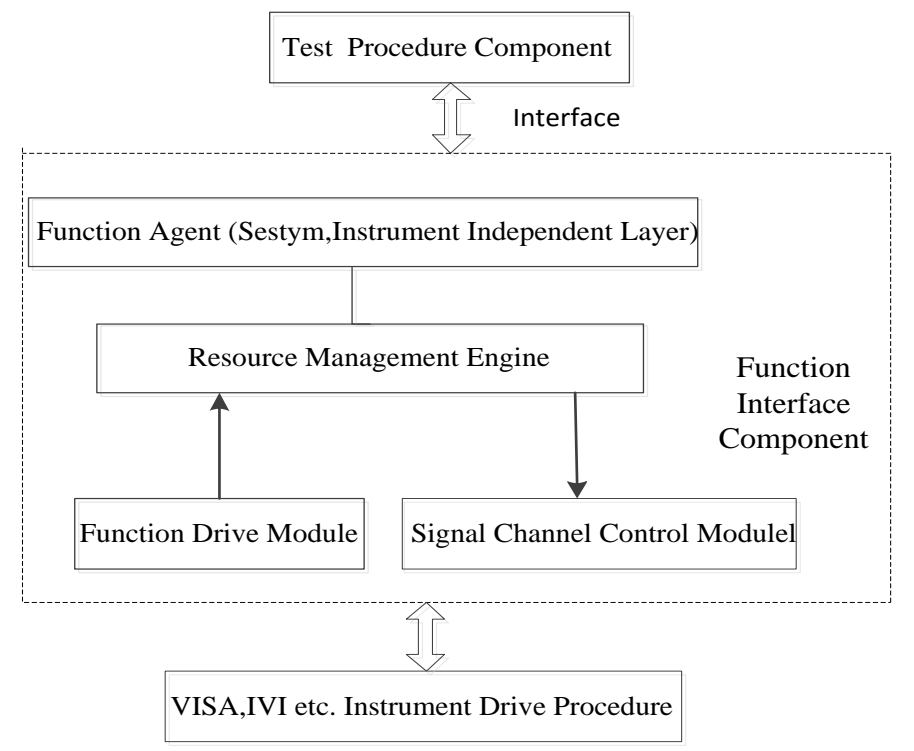

Figure 1 The Architecture of the Networked Function Interface Based on Component

\section{Function Agent Layer.}

The function agent layer gives an interpretive interface by test behavior facing test or excitation function. And test or excitation functions are realized as the membership .The object gets the information for testing by the means of test or excitation. The interface shields the function agent internal mechanism, not presenting any other information with the instrument. It describes the process on test flow and result decision. The development of TPS is fully made directing toward UUT interface. The instrument control has no relationship with test procedure. And it isolated with test system completely.

\section{Resource management Engine.}

The resource management engine provides services for automation resource allocation and switch path compute. It's the core of the whole modules. And it receives the function information delivered by the function agent module. It checks the rationality of TPS on the grounds of equipment, switch and adapter information. It completes the mapping from virtual resource into real 
resource, the automatic allocation of real resource and the optimal route search of signal channel, in accordance with test requirement, resource module information and optimal principle of resource utilize. And it calls correctly instrument function driver and signal channel control module, according to system resource allocation program.

\section{Function Drive Module.}

Control action of instrument and switch facing signal can be done by instrument function driver module of function interface and signal channel control module. The realized functions with all the instruments of test system will be classified by instrument function driver module. And the realized details are also neglected with specific function of each instrument. No matter which kind of instrument, there is the same function as early as the same function driver interface. When the instrument module is exchanged, there is no change with the interface of new instrument function driver module, so long as the same function. The new function driver is registered again with system resource module allocation tool. Then the original function driver is replaced. There is no change with function driver for the system, but it realizes the interchangeability of the instrument genuinely. The instrument driver module adopts the standard easily. It contains DAQ base, IVI standard, VPP driver procedure, bottom call and so on. The instrument function driver is designated by the instrument. It supports the flexible allocation from the instrument into the signal function. And it provides external interface for calling by resource management engine. There is a one to one correspondence with the interface information and function agency interface information. Thereby, it's more convenient for the information exchange between function agency layer and instrument function driver layer.

\section{Control Module of Signal Channel.}

The task of the control module of signal channel is to connect the electrical nodes among each signal node of the measurand and each test node of the system test resource. The signal channel control module is a kind of software packaging of switching function to the system switch resource. It achieves the specific function of the channel switch through the control switch of each kind of driver software interface of switch resource. And it provides the standardized software interface controlled easily for the resource management engine. The interface parameter just touches open the name of the UUT signal node with needed electrical connection. The control operation implementation of the specific channel control element is achieved in the module. The electrical connection of each signal node of ATS is put as the basic origin for the control to the signal channel from the test procedure. At last, there is no relationship with test procedure and the electrical connection of the system signal channel.

\section{Interchangeable Implementation}

The function interface component separates the instrument control from the test program completely, makes fully the test program face the signal. The ability of the test or the excitation with the signal is recognized as the basic unit, implicating the specific instrument. For instance, a signal generator can generates five kinds pumping signals. They are direct current, sine wave, square wave, triangle wave and arbitrarily wave. The instrument can be derived in using five function drive modules following the classification by signal excitation function. If D/A output can also generate the excitation signals above, it has the same type with function drive interface together with the signal generator. As the same, the instrument can be driven by five function drive modules. When the need of the system resource is the sine wave signal, it's all has the same excitation function with signal generator and D/A. In that case, it's possible to choose any instrument. It merely exposes function interface and shields, the difference with the specific instrument, to the test system, and establishes insulating layer between test program and the specific instrument control. Then, it made the instrument control and test program separate perfectly. And there is an assurance of the instrument interchangeability of TPS. 


\section{Conclusions}

In this paper, network function interface module based on module technology was presented to resolve using TPS, upgrading and replacing instrument conveniently, referenced from the module with IVI, IVI-MSS and IVI-Signal Interface. Module structure was introduced, and function agent layer, resource management engine, function drive module and control module of signal channel were introduced for the network function interface module based on module technology. Finally, Interchangeable was implemented.

\section{References}

[1] Geathers G E. The IVI foundation signal interface:a new industry standard[ Z] . IEEE, 2001:497 - 503.

[2] IVI Foundation IVI Signal Interface, Functional Specification, Rev. 0.3,IVI Foundation, October 2000.

[3] Tyler D. Java based automated test system management considerations for an open architecture for test [A]. Proc. AUTOTESTCON,San Antonio ,TX,1999:699 - 706.

[4] IVI Foundation,IVI-3.10: Measurement and Stimulus Subsystem(IVI-MISS) Specification, Revision 0.4,February 2001.

[5] EllisK, Delaney D, Gorringe, C. ATLAS2K-The framework for precise and extensible signal descript ions in modern ATS. AUTO TEST CON Proceedings, 2001[A ]. IEEE System s Readiness Techno logy Conference[C ]. 2001. 468-479.

[6] Cherfas A , ReevesW. ATLAS2000 signal and method classification and modeling [A ]. AUTO TESTCON ’98.IEEE Systems Readiness Technology Conference[C ]. 1998. 139-144. 